\title{
DESINFORMACIÓN, BULOS, CURACIÓN Y VERIFICACIÓN. REVISIÓN DE ESTUDIOS EN IBEROAMÉRICA 2017-2020.
}

\author{
Javier Guallar* \\ https://orcid.org/0000-0002-8601-3990 \\ Lluís Codina** \\ https://orcid.org/0000-0001-7020-1631 \\ Pere Freixa** \\ https://orcid.org/0000-0002-9199-1270 \\ Mario Pérez-Montoro**** \\ https://orcid.org/0000-0003-2426-8119
}

RECIBIDO: Julio 2020 / ACEPTADO: Agosto 2020 / PUBLICADO: Septiembre 2020

\begin{abstract}
Como citar: Guallar, Javier; Codina, Lluís; Freixa, Pere; Pérez-Montoro, Mario (2020). Desinformación, bulos, curación y verificación. Revisión de estudios en iberoamérica 2017-2020. Telos: revista de Estudios Interdisciplinarios en Ciencias Sociales, 22 (3), Venezuela. (Pp.595-613).

DOI: www.doi.org/10.36390/telos223.09
\end{abstract}

\section{RESUMEN}

El objetivo de este artículo es realizar una revisión de las investigaciones sobre desinformación en el ámbito de Iberoamérica entre 2017 y 2020. Para ello, se siguen las normas APA para revisiones y se analizan alrededor de 60 trabajos publicados en revistas indexadas de Iberoamérica, así como libros publicados sobre la temática. Los resultados se muestran agrupados en tres partes: En la primera se revisan los tres conceptos fundamentales relacionados con la desinformación: el propio término de desinformación, así como posverdad e infodemia. En la segunda, se estudian los principales productos de desinformación: noticias falsas, desórdenes informativos y bulos, atendiendo a sus tipos, temáticas, formatos, y canales. En la tercera parte se presentan las principales estrategias contra la desinformación, revisando los trabajos publicados de dos de ellas: la curación de contenidos y la verificación de hechos. Los autores más destacados sobre la temática a nivel cuantitativo son Magallón-Rosa con 6 trabajos, Ufarte-Ruiz con 4 y García-Marín con 3 trabajos. Asimismo, se pueden destacar por su análisis de la desinformación en el área de lberoamérica los estudios de Palau-Sampio (2018), Vizoso y Vázquez-Herrero (2019) y Rodríguez-Pérez (2020), por su análisis de las tipologías de bulos el trabajo de Salaverría et al (2020) y por las propuestas sobre curación los trabajos de

\footnotetext{
* Universidad de Barcelona. Centre de recerca en Informació, Comunicació i Cultura CRICC. Facultad de Información y Medios Audiovisuales, Barcelona, España. Correo electrónico: jguallar@ub.edu

** Universidad Pompeu Fabra. Facultad de Comunicación, Barcelona, España. Correo electrónico: Iluis.codina@upf.edu

*** Universidad Pompeu Fabra. Facultad de Comunicación, Barcelona, España. Correo electrónico: pere.freixa@upf.edu

**** Universidad de Barcelona. Centre de recerca en Informació, Comunicació i Cultura CRICC. Facultad de Información y Medios Audiovisuales, Barcelona, España. Correo electrónico: perez-montoro@ub.edu
} 
López-Borrull con colaboradores. Entre las conclusiones, se ha visto que el fenómeno de la desinformación es altamente poliédrico, pero que la sociedad tiene instrumentos para afrontarlo, como la curación y la verificación o fact cheking.

Palabras claves: Desinformación, posverdad, infodemia, bulos, noticias falsas, curación de contenidos, verificación, verificadores.

\section{Misinformation, hoaxes, curation and verification. Review of studies in Iberoamerica 2017-2020.}

\section{ABSTRACT}

The objective of this article is to carry out a review of misinformation research in the lberoAmerican area between 2017 and 2020. To do this, APA standards for reviews are followed and around 60 papers published in indexed journals in Ibero-America are analyzed, as well as books published on the subject. The results are shown grouped into three parts. In the first, the three fundamental concepts related to misinformation are reviewed: the term of misinformation itself, as well as post-truth and infodemic. In the second, the main misinformation products are studied: false news, information disorders and hoaxes, according to their types, themes, formats, and channels. In the third part, the main strategies against misinformation are presented, reviewing the published works of two of them: content curation and fact checking. The most outstanding authors on the subject at a quantitative level are Magallón-Rosa with 6 works, Ufarte-Ruiz with 4 and Garcia-Marín with 3 works. Likewise, the studies by Palau-Sampio (2018), Vizoso and Vázquez-Herrero (2019) and Rodríguez-Pérez (2020) can be highlighted for their analysis of misinformation in the Ibero-American area; for their analysis of the typologies of hoaxes the work of Salaverría et al (2020) and for the proposals on curation the works of López-Borrull with collaborators. Among the conclusions, it has been seen that the phenomenon of misinformation is highly polyhedral, but that society has instruments to deal with it, such as curation and verification or fact cheking.

Keywords: Misinformation, post-truth, infodemic, hoaxes, fake news, content curation, verification, fact checking, fact checkers.

\section{Introducción}

Se presenta una revisión de los estudios recientes en torno a la desinformación en el ámbito de lberoamérica (en la acepción del término que contempla los países de lenguas latinas de América junto a España y Portugal). Se revisan para ello las aportaciones de los principales estudios publicados entre 2017 y 2020 en torno a tres aspectos relacionados entre sí:

1) En primer lugar, la desinformación en sí como fenómeno, así como los conceptos relacionados de posverdad y de infodemia.

2) A continuación, los principales productos o manifestaciones de la desinformación, es decir, los bulos, desórdenes informativos y noticias falsas (fake news), analizando sus tipos, temáticas, formatos y canales. 
3) Por último, se muestran las principales estrategias de respuesta a la desinformación, dedicando especial atención a dos de ellas, la curación o curaduría y la verificación (fact checking).

Para cada bloque se presentan y discuten los términos más empleados y relacionados, analizando las principales aportaciones de la bibliografía de ámbito iberoamericano con ejemplos o manifestaciones relevantes. Como enfoque para este trabajo, se han adoptado las recomendaciones APA en su $7^{a}$ edición (American Psychological Association, 2020) sobre artículos de revisión (Literature Review Articles), en las cuales se recomienda que los autores de un artículo de revisión definan con claridad el problema, sinteticen o resuman investigaciones previas para informar a los lectores de la situación del problema, identifiquen relaciones en la bibliografía analizada y sugieran posibles pasos en la solución del problema. En lo que sigue, se ha procurado seguir las pautas que recomienda APA para esta clase de trabajos.

\section{Desinformación, posverdad e infodemia}

En este apartado se realiza una aproximación a los tres términos más empleados para aludir al fenómeno que estudiamos a partir de lo que ha quedado establecido la bibliografía reciente.

\section{Desinformación}

Es el término sobre el que existe más consenso para definir el fenómeno al que estamos asistiendo en años recientes a nivel global, y que cuestiona la veracidad de muchas de las informaciones y contenidos a los que estamos expuestos y que consumimos a diario los ciudadanos en todo el mundo.

Una definición de desinformación comúnmente aceptada es la propuesta por la Comisión Europea en 2018, para referirse al contenido "falso, inexacto o engañoso [...] diseñado, presentado y promovido intencionalmente para causar daño público o beneficios particulares" (European Commission, 2018), o también, añade Del-Fresno-García: para engañar o "alterar la percepción de forma intencional de grandes grupos de personas o sociedades e influir en su comportamiento (político, económico, ideológico...)" (Del-Fresno-García, 2019, p. 3).

Vemos por tanto que existirían tres grandes tipos de contenidos que producen desinformación: los contenidos falsos, los inexactos y los engañosos. Pero tan importante como estos tipos de productos, sobre los que volveremos en la siguiente sección, será el motivo o la intención que hay detrás de ello. Como vemos, o bien causar un daño público, o bien producir unos beneficios particulares, o bien engañar 0 alterar la percepción de las personas e influir en su comportamiento (o varias de estas cosas a la vez). Habría por tanto una intencionalidad determinada, de manera que esa manipulación, falsificación, o transformación (en diversos grados 0 de distintas maneras), de la realidad, son instrumentos para conseguir los fines de causar daño, de obtener beneficios o de influir en el comportamiento de las personas, unos fines que usualmente se ocultan y permanecen invisibles 0 al menos no se explicitan para el público destinatario.

Aunque no se trata de un fenómeno nuevo (para un resumen de su evolución histórica, se puede consultar Parra Valero y Oliveira, 2018), lo novedoso del mismo es la gran dimensión alcanzada en nuestros días: "la era de los datos masivos (big data), ha hecho también posible las mentiras masivas" (Guallar, 2018). La dimensión masiva hace que el problema sea en la 
actualidad mucho más importante de lo que nunca lo haya sido en el pasado. Una percepción muy extendida, además, es que el problema, lejos de estar en vías de solución, va a ir en aumento. Una muestra de esta perspectiva (de tintes catastrofistas) es el conocido informe de la consultora Gartner que alertaba en 2017 que en 2022 el público llegará a consumir más noticias falsas que verdaderas (Panetta, 2017).

Las razones que pueden explicar este fenómeno de la desinformación son múltiples, por lo que los investigadores hablan de un problema que tiene una raíz multidimensional (Wardle; Derakhshan, 2017; Ireton; Posseti, 2018; Aparici; García-Marín, 2019), si bien los motivos se pueden clasificar en dos grandes grupos. Por una parte, los avances tecnológicos y las propias características de la web social, que favorecen la expansión de cualquier falsedad de manera instantánea y global con un alcance potencialmente masivo. Por otra parte, diversas motivaciones psicológicas, como los procesos de disonancia cognitiva o los sesgos de confirmación, entre otros (García-Marín, 2020), que hacen que las personas no solo crean con facilidad informaciones falseadas, sino que incluso les puedan dar más credibilidad que a las verdaderas.

Vale la pena detenerse también en otros dos términos estrechamente relacionados con la desinformación, que aportan matices específicos y complementarios: posverdad e infodemia.

\section{Posverdad}

La extensión de situaciones y procesos de desinformación a la que estamos aludiendo, ha hecho emerger con fuerza otro término, posverdad (post-truth), que fue declarado palabra del año en 2016 por el Oxford English Dictionary. La definición de Oxford University Press, una de las más empleadas, considera posverdad "las circunstancias en las cuales los hechos objetivos son menos influyentes para moldear la opinión pública que apelar a la emoción y las creencias personales" (Oxford University Press, 2016). Otras definiciones presentan matices diferentes, pero como señalan Rodrigo-Alsina y Cerqueira, todas ellas concuerdan "en la influencia de las emociones y las creencias en las personas y en la opinión pública" (Rodrigo-Alsina; Cerqueira, 2019, p. 226). Así pues, "la era de la información y la comunicación ha resultado ser la de la emoción" (Aparici; García-Marín; Rincón-Manzano, 2019). Esto implica que la subjetividad se impone sobre la objetividad; y que la valoración, visión o emoción personal pueden llegar a ser más importantes que la propia realidad. Llevado el planteamiento al extremo, se menospreciaría la realidad objetiva para creer en una nueva realidad en la cual "aquello que se siente, no sólo se siente; es, además, la verdad" (Del-Fresno-García, 2019, p. 3).

Capilla (2019), va más allá y en un estudio en el que profundiza en el concepto de posverdad a partir del uso que los medios de comunicación hacen del mismo, concluye que: "(a) la posverdad es un concepto de la comunicación política que plantea un cambio en la relación que la sociedad tiene con la verdad; $y$ (b) es un término con un sesgo político que se utiliza para designar a un oponente percibido como enemigo del modelo de sociedad" (Capilla, 2019). Por último, para algunos autores, este fenómeno solo se explica por su estrecha vinculación con la pérdida de influencia de los medios de comunicación a favor de las redes sociales: "las redes sociales mandan (...) la sociedad de la posverdad ya no es una responsabilidad de los medios, como en el siglo XX" (Marcos Recio; Sánchez Vigil; Olivera Zaldua, 2017), en una situación que no sería sino una manifestación más de la actual "decadencia de la esfera pública" (Álvaro Sánchez, 2019). 


\section{Infodemia}

Este último término, se ha expandido con gran rapidez en 2020, desde que el 15 de febrero la Organización Mundial de la Salud declarara como prioridad la lucha contra la proliferación de noticias e informaciones falsas o engañosas en relación con la pandemia de la Covid-19, calificando esta situación de "infodemia" (WHO, 2020). Así, la infodemia, que vendría ser una acentuación de una situación de desinformación, literalmente una "epidemia de desinformación", sería el nivel extremo del fenómeno al que estamos aludiendo, y se suele identificar con un periodo de tiempo más o menos acotado y en relación con un hecho o suceso concreto, generalmente empleado en un contexto de crisis sanitaria. En este sentido, se puede considerar que la desinformación a nivel global en torno al coronavirus Covid19 en 2020 ha alcanzado estos niveles de infodemia, aunque estrictamente en otros momentos del pasado también se haya hablado de infodemias (García-Marín, 2020). Asimismo, una variante del término la emplea la UNESCO en sus informes, al hablar de "desinfodemia" (Posetti; Bontcheva, 2020a; Posetti; Bontcheva, 2020b).

Una vez situado el fenómeno en su dimensión conceptual y global, veamos a continuación sus manifestaciones (bulos y desórdenes informativos) así como las posibles acciones de respuesta.

\section{Manifestaciones de la desinformación: tipos, temáticas, formatos y canales}

A continuación, se presentan los términos más comúnmente empleados para identificar las manifestaciones de la desinformación, esto es, noticias falsas, desórdenes informativos y bulos, y se muestran los resultados de algunas de las investigaciones recientes de ámbito iberoamericano en torno a sus tipos, temáticas, formatos y canales de diseminación.

\section{Noticias falsas, desórdenes informativos y bulos}

Los términos más empleados para referirnos a las manifestaciones o productos que generan desinformación son seguramente estos tres: noticias falsas (fake news), desórdenes informativos y bulos.

Noticias falsas (fake news) es la expresión más extendida tanto en la bibliografía académica como a nivel popular para aludir a estas manifestaciones concretas de la desinformación. Fue elegida palabra del año en 2017 por el Collins Dictionary, que las define como "información falsa, a menudo sensacional, difundida bajo el disfraz de noticia", siendo una expresión que aparece "casi indisolublemente ligada" a las de desinformación y posverdad (Rodríguez-Ferrándiz, 2019). El Oxford Dictionary (2019), las define como "noticias que transmiten 0 incorporan información falsa, fabricada o deliberadamente engañosa, o que se caracterizan o acusan de hacerlo" y sitúa el inicio de la popularización del término en las elecciones presidenciales norteamericanas de 2016.

Los detractores de su uso, con argumentos como que una noticia si es falsa ya no es una noticia, instan a utilizar preferentemente los otros dos, más precisos, o bien el genérico de desinformación (véase, por ejemplo, Rodríguez-Pérez, 2019). Así, con relación al término de noticias falsas, se da la circunstancia de que, por un lado, en cualquier discusión sobre el tema es casi inevitable traer este concepto (como se ha hecho en este trabajo), pero por otro, desde diferentes instancias, como la Unesco (Ireton y Posetti, 2018) se recomienda no usar esta 
expresión. Esta última recomendación está dirigida sobre todo a periodistas, curadores y divulgadores, y el motivo que alega la Unesco es su uso interesado por parte de algunos gobiernos. En su lugar, proponen que se usen los conceptos de desinformación o cualquier otro relacionado, como el de desórdenes informativos, del que nos ocupamos a continuación.

Desórdenes informativos (Information disorders) sería la expresión con una concepción más amplia (la más diversa) de las tres, pues englobaría todas las posibles variantes. Para Del-Fresno-García (2019), son "producciones intencionales cuya estrategia consiste en la fabricación de la duda y falsas controversias con el fin de conseguir beneficios económicos o ideológicos".

Bulos, por último, es un término clásico, que en el contexto actual ha ganado en aceptación entre las investigaciones más recientes en detrimento del de fake news. Salaverría et al (2020), firmes defensores de su utilización, los definen así: "todos aquellos contenidos falsos que alcanzan difusión pública, fabricados intencionadamente por múltiples motivos, que pueden ir desde la simple broma o parodia, hasta la controversia ideológica, pasando por el fraude económico". Estos autores advierten, no obstante, los límites de los bulos son difusos y que si bien en algunas ocasiones son claramente premeditados, en otras no son más que exageraciones, malinterpretaciones o confusiones. Hagamos a continuación un repaso de los principales tipos, formatos, temáticas y canales de desinformación a partir de lo que ha quedado establecido en las investigaciones recientes.

\section{Tipos}

Es difícil realizar una categorización precisa que englobe todas las tipologías de bulos, por lo que nos vamos a referir solamente a algunas de las propuestas, las que consideramos que han alcanzado una categorización más amplia o integradora de las variantes observadas. Aparici, García-Marín y Rincón-Manzano (2019), en un estudio sobre la desinformación en torno al conflicto catalán de octubre de 2017, categorizan cinco tipos de bulos:

- Falsa atribución: relacionar imágenes de otros contextos, lugares y/o momentos con los hechos actuales

- Exageración de los hechos: informaciones que no son completamente falsas, pero se exageran para mostrar de manera reforzada un argumento

- Manipulación de imágenes: fotografías en las que se añaden elementos inexistentes para reforzar un mensaje

- Invención de los hechos (categoría que los autores identifican con las fake news): contenidos enteramente falsos e inventados que utilizan tácticas de guerrilla de marketing 2.0, como bots automáticos y la suplantación de identidad

- Suplantación de identidad (counterfeit): una subcategoría específica de la anterior, que consiste en crear páginas o perfiles falsos en redes sociales que imitan la imagen de marcas corporativas o de personas reales

Por su parte, Salaverría et al (2020), analizando un contexto más amplio (no solo político), establecen cuatro grandes tipos de bulos: bromas, exageraciones, descontextualizaciones y engaños, de menor a mayor gravedad:

- Broma: difusión de información falsa, con un fin burlesco, paródico, satírico o caricaturesco. 
- Exageración: contenido que tiene cierto vínculo con la verdad, pero traspasa los límites de lo verdadero y entra en el terreno de la falsedad.

- Descontextualización: Bulo que utiliza hechos o declaraciones reales en un contexto deliberadamente falso o tergiversado.

- Engaño: falsificación absoluta, en la que se fabrican contenidos con la intención de hacer creer a la ciudadanía declaraciones o hechos falsos.

\section{Temáticas}

En cuanto a temáticas, si bien los resultados de los estudios existentes son difíciles de comparar por cuanto analizan contextos temporales diferentes y en canales o países distintos, sí se pueden ver algunas tendencias generales. A nivel global, la información política ha sido el ámbito más abundante tradicionalmente en desinformación, junto a otros temas también recurrentes como los bulos sobre migración (con enfoque racista) o los bulos sobre ciencia, si bien en 2020, con la extensión de la pandemia de la Covid-19, los bulos con contenidos de salud han crecido notablemente.

Así por ejemplo, Bernal-Triviño y Clares-Gavilán (2019), clasifican los bulos en 2018 en estas categorías y porcentajes: política (35\%), personajes (15\%), inmigración o racismo (12\%), género $(10 \%)$ y ciencia $(9 \%)$. Y si vamos a trabajos más recientes, de 2020 , vemos que Salaverría et al (2020), establecen tres grandes categorías: Ciencia y Salud; Política y Gobierno; y Otros, con una distribución bastante homogénea de los bulos entre las tres; Pozo-Montesa y León-Manove (2020), señalan que la temática predominante de los bulos es la política y las falsas atribuciones a instituciones; Sánchez-Duarte y Magallón-Rosa (2020) distinguen cuatro temáticas en relación con la Covid-19: contagios, prevención, medidas adoptadas contra la pandemia y otros (seguridad, origen del virus, predicciones, etc.) y García-Marín (2020), también sobre la Covid-19, encuentra temáticas bastante similares al estudio anterior.

Por último, en el informe de la UNESCO de Posetti y Bontcheva (2020a), se señalan como principales temas de desinfodemia los siguientes:

- Orígenes y propagación del coronavirus y de la enfermedad

- Ciencia médica: síntomas, diagnóstico y tratamiento

- Estadísticas falsas y engañosas

- Impactos sobre la sociedad y el medio ambiente

- Impactos económicos

- Desacreditación de periodistas y medios de comunicación

- Politización

- Contenido promovido por celebridades que buscan ganancias económicas fraudulentas.

\section{Formatos}

Este informe de Posetti y Bontcheva (2010 a) para la UNESCO identifica cuatro formatos clave de desinformación:

- Narraciones y memes emotivos

- Sitios Web e identidades inventadas

- Imágenes y videos fraudulentamente alterados, inventados o descontextualizados 
- Infiltración y campañas de desinformación planificadas

En otros estudios, como en el de Salaverría et al (2020), se destaca que el formato predominante sigue siendo el texto, a menudo en combinación con otros formatos multimedia, y llama la atención que no se localizan casos de formatos tan peligrosos como los "deep fakes" basados en la creación de imágenes falsas mediante inteligencia artificial.

\section{Canales}

Si distinguimos tres grandes tipos de canales en la difusión de contenidos de desinformación: los medios de comunicación, las redes sociales abiertas (como Twitter, Facebook o Instagram) y las redes sociales cerradas y servicios de mensajería (como Whatsapp, Telegram o grupos de Facebook), los resultados de diversos estudios vienen a coincidir en torno al gran peso que tiene este último grupo en la distribución de la desinformación, aunque también tiene una presencia apreciable el segundo grupo, siendo mucho más escasa la presencia de bulos en el tercer grupo, los medios de comunicación. A este respecto, concluyen Salaverría et al (2020, p. 11): "WhatsApp se ha revelado, en efecto, como la plataforma donde los bulos se diseminan en mayor cantidad y con mayor alcance. Con todo, también se advierte una considerable difusión de contenidos falseados en redes sociales abiertas, como Twitter".

Diversos estudios realizados sobre la desinformación en WhatsApp abundan en esta idea: Así, Canavilhas, Colussi y Moura (2019) estudian la desinformación en grupos de WhatsApp durante la campaña de las elecciones presidenciales de Brasil de 2018, con un estudio de campo propio (no basado en desmentidos de fact checkers) y encuentran que el 60 $\%$ de los 472 posts analizados, compartidos en grupos de Whatsapp, contienen información total o parcialmente falsa. Como señalan los autores, "los hallazgos corroboran la existencia de un círculo de desinformación entre los usuarios de WhatsApp" (Canavilhas; Colussi; Moura, 2019).

\section{Respuestas a la desinformación: marco general, curación y verificación}

En este apartado presentamos diferentes estrategias de respuesta a la desinformación, y lo hacemos, ofreciendo en primer lugar un marco general, para a continuación, detenernos en la literatura reciente acerca de dos de ellas, que se pueden situar entre las más importantes: la curación y la verificación.

\section{Marco general}

Los informes de la UNESCO (Ireton; Posetti, 2018; Posetti; Bontcheva, 2020a; 2020b) proporcionan un marco general para situar los diferentes tipos de respuestas y de medidas contra la desinformación, desde respuestas políticas, económicas y legislativas a las educativas 0 a actividades concretas como el monitoreo y la verificación y las actividades curatoriales. Dicho marco se puede considerar como una estrategia global contra la desinformación, dentro de la cual se pueden enmarcar las diferentes acciones, necesariamente como complementarias entre sí.

Así, un grupo de medidas contra la desinformación es el que se refiere a establecer unos sistemas de regulación eficaces, así como de procedimientos de actuación contra los sitios donde se publique información falsa. Una revisión de algunas de estas iniciativas se puede consultar en Ramón Fernández (2020). Más allá del frente regulador, el papel que adoptan las administraciones en general y los gobiernos en particular es por supuesto clave. 
En el caso de la pandemia de la Covid, diversas investigaciones recientes así lo han constatado. Por ejemplo, en el caso español, los bulos sobre el coronavirus formaron parte destacada de los mensajes del presidente del Gobierno español en sus comparecencias públicas durante el decreto del estado de alarma en marzo y abril de 2020, "advirtiendo a la población del peligro de compartir información falsa y apelando a que sólo se recurriese a fuentes oficiales y fiables" (Castillo-Esparcia; Fernández-Souto; Puentes-Rivera, 2020, p. 19). Más allá de las medidas concretas, una estrategia más de fondo debe incluir una profunda alfabetización mediática y digital de los ciudadanos (Lotero-Echeverri; Romero-Rodríguez; Pérez-Rodríguez, 2018).

Otro grupo de medidas esenciales contra la desinformación son las que se deben realizar desde las propias plataformas de redes sociales donde, como se ha visto, se difunden la gran mayoría de bulos. Catalán-Matamoros (2020) refiere algunas de ellas: Twitter, YouTube y Whatsapp han reforzado sus filtros de verificación para reducir la circulación de información falsa en sus plataformas, incluyendo en algunos casos la retirada de bulos, y colaboran, para el caso de la Covid, con la Organización Mundial de la Salud y las autoridades sanitarias de diferentes países para favorecer el acceso a información fiable sobre la pandemia. Un ejemplo de ello sería la iniciativa de Facebook del Centro de Información sobre el Coronavirus (Facebook, 2020).

Otro frente necesario, es el de la investigación y la Academia. En cierta medida, un artículo como el presente estaría en esta línea que autores como Catalán-Matamoros defienden con vehemencia: desde la Academia es imprescindible contribuir analizando el particular contexto socio-cultural en el que vivimos, "gobernado por el declive de expertos y especialistas, el aumento de políticos populistas sin perfil científico, y de manera más profunda, por la psicología social de las emociones, valores y creencias" (Catalán-Matamoros, 2020, p. S6).

Dos profesiones, al menos, se han visto muy directamente interpeladas por la extensión generalizada de la desinformación, siendo constantes en la bibliografía reciente las manifestaciones de esta inquietud y preocupación, en referencia a cuál debería ser la actuación de los profesionales del periodismo (Mayoral; Parratt; Morata, 2017; Marzal-Felici; CaseroRipollés, 2017; Nigro, 2018; Blanco-Herrero; Arcila-Calderón, 2019; Rodríguez-Fernández, 2019; Sánchez de la Nieta Hernández; Fuente Cobo, 2020) y de la información y documentación (Caridad-Sebastián et al, 2018; López-Borrull; Vives-Gràcia; Badell, 2018). Vinculadas estrechamente con estas profesiones, dos actividades y/o estrategias fundamentales en la lucha contra la desinformación, son la curación y la verificación. Vamos a referirnos a ellas en la parte final de este trabajo.

\section{Curación (o curaduría) de contenidos}

Las actividades y servicios o productos curatoriales, es decir, de selección curada (esto es, con aportación de valor y contexto) de fuentes de información y contenidos fiables y de calidad, aparecen destacados en el reciente informe de la UNESCO de Posetti; Bontcheva, (2020b), como uno de los elementos clave en la lucha contra la desinfodemia. Los curadores, bibliotecarios, documentalistas y profesionales de la información en general tienen en este sentido la función y la responsabilidad de proveer de información de calidad a la ciudadanía contra la desinformación, desde los diferentes proyectos, servicios o unidades de información en los que se encuentren. En este sentido, López-Borrull, Vives-Gràcia y Badell (2018), 
consideran que la desinformación, más allá de una amenaza, constituye una oportunidad para estos colectivos profesionales.

En el sector de las bibliotecas y de la documentación en general, varios estudios enfatizan tanto el papel que ya cumplen como su potencial para ampliarlo en cuanto a actividades curatoriales contra la desinformación, tales como la selección de recursos, la elaboración de guías de lectura, la gestión de las colecciones bibliotecarias, o el apoyo a la docencia y la formación de usuarios en el uso de la información (Caridad-Sebastián, et al. 2018; López-Borrull; Vives-Gràcia; Badell, 2018; Martínez-Cardama; Algora-Cancho, 2019). En todos estos frentes se han llevado a cabo diversas iniciativas bibliotecarias y del sector de la información, algunas de ellas impulsadas por instituciones y asociaciones nacionales e internacionales, como las promovidas por la International Federation of Library Associations and Institutions (IFLA). Uno de los puntos fuertes del sector es, en palabras de López-Borrull, VivesGràcia y Badell (2018, p. 1354), que "las bibliotecas tienen una de las cosas más preciadas en el mundo: una marca respetada y en la que los usuarios confían".

En línea con estas acciones, López-Borrull y Ollé (2019), proponen en un trabajo reciente, en el que adaptan el paradigma de la curación de contenidos a la lucha contra la fake science, que las bibliotecas amplíen su papel en la alfabetización de la información mediática a los contenidos de tipo científico.

Otro ámbito profesional en el que documentalistas y curadores ejercen su función contra la desinformación es el propio sector de los medios de comunicación. En este terreno, los curadores, ya sean periodistas o documentalistas u otros profesionales, ejercen esa función curatorial de filtrado de información veraz a la que aportan sentido y contexto (Guallar; Codina, 2018; Codina; Guallar, 2019; Guallar; Cornet, 2020). Este aspecto enlaza con el siguiente apartado, estrechamente relacionado, y que está siendo el de mayor protagonismo en la lucha contra la desinformación: la verificación de informaciones.

\section{Verificación y fact checking}

La verificación no es en absoluto una novedad para los medios de comunicación, pues ha sido una práctica tradicional asociada al buen periodismo e indisolublemente vinculada a la especialidad de la documentación periodística (Redondo, 2018; Guallar; Cornet, 2020). Pero, aunque siempre haya estado presente entre las funciones esenciales de los profesionales del periodismo, la creciente preocupación por la desinformación la ha situado en un plano de ascendente protagonismo que no siempre había tenido anteriormente. Existen diversas publicaciones y manuales actualizados sobre su empleo, entre los que se puede destacar en castellano el manual de Redondo (2018): "Verificación digital para periodistas. Manual contra bulos y desinformación internacional". Además del uso genérico de la verificación que todo buen periodista o documentalista de prensa debe tener muy presente, han surgido en todo el mundo numerosos proyectos especializados en ello, como réplica precisamente a la extensión del fenómeno de la desinformación. Así, este movimiento de fact checking o de verificación de hechos, a través de numerosas plataformas a nivel global, ha llegado a ser considerado "la variante más importante del periodismo en la era digital", en palabras del fundador de la organización pionera Politifact Bill Adair (citado por López Pan; Rodríguez Rodríguez, 2020). En consonancia, las plataformas especializadas en fact checking han despertado un enorme interés en la investigación sobre desinformación en los años recientes, por lo que existen ya numerosísimos estudios sobre ellas, seguramente más que de cualquier otro aspecto de los que 
analizamos en este trabajo. Si bien un importante número de dichos trabajos son de ámbito estadounidense 0 a nivel mundial, hay también bastantes de interés de ámbito iberoamericano tanto a nivel de conjunto de varias plataformas de verificación, como de estudios de caso de plataformas concretas.

El fact checking es, según Amorós (2018), de todas las herramientas existentes, la mejor para contrarrestar la desinformación y las noticias falsas, y ha sido considerado por algunos autores como un nuevo género periodístico (Rodríguez-Pérez, 2020). Surge como tal en Estados Unidos en los primeros años del siglo XXI, aunque hay precedentes anteriores, estrechamente vinculado a la fiscalización de las falsedades en la información política, y despega rápidamente gracias a algunas marcas muy exitosas que generan tendencia, como la ya citada Politifact, fundada en 2007, o secciones especialidades en fact checking en medios como los famosos "pinochos" del Washington Post (Guallar, 2011; López-Pan; RodríguezRodríguez, 2020). Ese impulso inicial ha aumentado en los últimos años, en buena parte gracias a las actuaciones controvertidas del presidente Trump, constituyéndose así en referente para las organizaciones de fact checking del resto del mundo. Véase, por ejemplo, un estudio reciente en Magallón-Rosa (2018a).

La influencia estadounidense llegó en pocos años a Iberoamérica, siendo el referente inicial en la región la plataforma argentina Chequeado, nacida en 2010 y que en 2015 obtiene el premio Gabriel García Márquez de la Fundación para el Nuevo Periodismo Iberoamericano (FNPI), en el apartado de Innovación. Para seguir el surgimiento y evolución de algunas de estas iniciativas en lberoamérica se puede consultar Echevarría (2017).

Cubriendo un ámbito cercano al que seguimos en este trabajo, Vizoso y VázquezHerrero (2019) analizan todas las iniciativas de fact checking existentes en español a partir de la base de datos del Duke Reporters' Lab y localizan 19 plataformas, en lo que se puede considerar el estudio más exhaustivo de los que podemos reseñar para el ámbito iberoamericano. Considerando solo las 14 iniciativas activas, analizan Chequeado (Argentina), Chile Check y El Polígrafo (Chile), Colombia Check y Detector de mentiras (Colombia), El Objetivo, La Chistera, Maldito Bulo y Polétika (España), Detector de mentiras (Estados Unidos), Con Pruebas (Guatemala), El Sabueso (México), UY Check (Uruguay) y Cotejo (Venezuela). De estas plataformas estudian, entre otros aspectos, su sistema de trabajo, sus métodos de verificación y la profesión de sus miembros (son mayoría los periodistas si bien tienen cabida otros profesionales como analistas de datos y expertos en informática y estadística). En cuanto a las fórmulas para dar a conocer la veracidad o falsedad de las informaciones analizadas, predominan las explicaciones textuales, así como las escalas de verificación entre los términos "verdadero" y "falso", y en algunos casos, también se emplean escalas visuales y cromáticas.

Por su parte, Palau-Sampio (2018), analiza las plataformas de fact checking de Latinoamérica. A partir de la misma base de datos del Duke Reporters' Lab localiza 17 iniciativas, y descartando las inactivas, analiza 11 de ellas, buena parte de las cuales coinciden con las analizadas en el estudio anterior: Chequeado (Argentina), Truco, Aos Fatos y Agéncia Lupa (Brasil), Colombia Check y Detector de mentiras (Colombia), Con Pruebas (Guatemala), El Sabueso (México) y UY Check (Uruguay). De su análisis, en buena parte coincidente con el de Vizoso y Vázquez-Herrero (2019), se pueden destacar distintos aspectos de interés: la constatación del relevante papel de Chequeado en el desarrollo de la verificación en la región, ya que la plataforma argentina asesoró la puesta en marcha de otras, como las analizadas de 
Colombia, México y Uruguay; la utilización de fuentes para la verificación, en lo que también destaca Chequeado por ser la plataforma que consulta un promedio de fuentes superior $(6,8)$ y la tipología en la gradación comparativa de veredictos:

- verdadero, cierto... (verdad)

- cierto con matices, verdadero, pero... (casi verdad)

- exagerado, inflado, ridículo, engañoso, impreciso... (indefinido)

- insostenible, casi falso (casi falso)

- falso (falso)

- no se puede probar... (no evaluable)

En esta línea, el trabajo más reciente publicado de ámbito iberoamericano, RodríguezPérez (2020), analiza con resultados similares los que considera como los seis principales medios de verificación sudamericanos y españoles: Chequeado (Argentina), Colombia check y La Silla Vacía (Colombia), Ecuador Chequea (Ecuador) y Maldita y Newtral (España).

Si atendemos a estudios de caso por plataformas o países, encontramos una gran variedad de ellos de ámbito español. Empezando por estudios de conjunto sobre plataformas en España, Ufarte-Ruiz, Murcia-Verdú y Ruiz-Murcia (2018), señalan que en ese momento existen un total de diez proyectos de verificación; seis de ellos son secciones de medios de prensa o televisión (de El país, El confidencial, La sexta...) y 4 son independientes: Maldita hemeroteca, Maldito bulo, Mala prensa y Miniver. Más recientemente, López Pan y Rodríguez Rodríguez (2020) hacen un estudio global de las plataformas existentes, y muestran su génesis, característica y evolución, destacando que existen tres independientes: Maldita, Newtral y Verificat, además de secciones y blogs en medios periodísticos, así como iniciativas cívicas especializadas, como Salud sin bulos. También recientemente, Ufarte-Ruiz, Anzera y Murcia-Verdú (2020), hacen un estudio comparativo de plataformas independientes españolas e italianas, y en lo que se refiere a España analizan el método de verificación de informaciones, el modelo de negocio y los mecanismos de interactividad de Maldito bulo (submarca de Maldita) y de Newtral. Y por último, Salaverría et al (2020), en un estudio muy completo al que ya se ha hecho referencia, analizan los bulos desmentidos por las tres plataformas españolas incluidas en la International FactChecking Network (IFCN): Maldita, Newtral y EFE Verifica.

En cuanto a estudios de caso sobre plataformas concretas, destacan en número los dedicados a Maldita. Así, Magallón-Rosa (2018), y Bernal-Triviño y Clares-Gavilán (2019), analizan el sistema de funcionamiento de Maldito Bulo y de Maldita respectivamente mediante el análisis de los primeros 3000 tweets de Maldito Bulo en el primer caso y de entrevistas a los responsables de Maldita, así como análisis de contenido de 568 bulos desmentidos en 2018, en el segundo caso.

También sobre Maldita, Sánchez-Duarte y Magallón-Rosa (2020), analizan 166 bulos reportados por esta organización a la plataforma colaborativa Latam Chequea entre febrero y abril de 2020, y por último, Molina-Cañabate y Magallón-Rosa (2019, 2020), analizan en sendos estudios dos de sus submarcas especializadas en los bulos sobre migración/racismo y sobre ciencia, respectivamente: Maldita Migración y Maldita Ciencia. En lo que se refiere a estudios sobre Newtral, Pozo-Montesa y León-Manove (2020), analizan 104 bulos sobre Covid-19 desmentidos por Newtral entre el 14 de marzo y el 4 de mayo de 2020, subrayando algunas cuestiones ya observadas en otros estudios como que el canal principal para su difusión ha sido 
WhatsApp, y que destacan las falsas atribuciones a instituciones para intentar engañar a la ciudadanía.

Asimismo en España, Ufarte-Ruiz y Murcia-Verdú (2018), hacen un estudio de caso de la plataforma pionera en España y ya desaparecida Miniver, mostrando sus características y explorando su modelo de negocio. Y en lo que es bastante menos habitual en la bibliografía, Palomo y Sedano (2018) analizan como estudio de caso la sección de verificación de un diario: B de Bulo, del diario Sur y recientemente, Ufarte-Ruiz, Galletero-Campos y López-Cepeda (2020) analizan los proyectos de verificación de dos televisiones públicas en España: RTVE Verifica, de RTVE y Coronabulos, de la televisión vasca, EiTB.

Por otra parte, y ya finalizando este recorrido, son mucho más escasos los estudios de caso sobre alguna plataforma de verificación de otros países de la región. Entre ellos, podemos destacar que Vázquez-Herrero, Vizoso y López-García (2019), al estudiar plataformas de verificación de todo el mundo, seleccionan la argentina Chequeado como la más representativa de Iberoamérica, observando la estructura de sus piezas de verificación, los formatos de verificación y el sistema de difusión a través de Twitter y Facebook. Asimismo, Magallón-Rosa (2019), estudia el uso de Twitter por parte de Verificado México durante la campaña electoral mexicana de 2018, un proyecto muy interesante por cuanto reunió un equipo de más de 90 medios de comunicación y organizaciones que se asociaron para verificar la información electoral entre el 29 de marzo y el 27 de junio de 2018 . El estudio identifica los momentos concretos que aceleran la verificación, que son los debates electorales y la jornada electoral y distingue los tipos de bulos según los periodos de precampaña, campaña y postcampaña. $Y$ finalmente Seibt (2020), analiza el proyecto Truco nos Estados, de la Agência Pública, durante las elecciones de 2018 en Brasil, desde el punto de vista de las aportaciones que ofrece el fact checking a los cambios estructurales en el periodismo.

\section{Conclusiones}

Este trabajo se alinea con las recomendaciones de los principales organismos internacionales que luchan contra la desinformación y la infodemia, aportando un trabajo de revisión que puede ser útil para investigadores, profesionales y estudiosos del tema.

Los trabajos de revisión, como es este caso, además ayudan a establecer desarrollos futuros de tipo interdisciplinar, pues ponen sobre la mesa el estado de la cuestión, a partir del cual equipos interdisciplinares tienen una base común.

Se ha podido constatar que en el periodo estudiado, los autores más destacados sobre la temática a nivel cuantitativo son Magallón-Rosa con 6 trabajos, Ufarte-Ruiz con 4 y GarcíaMarín con 3 trabajos; asimismo, se pueden destacar por su análisis de la desinformación en el área de Iberoamérica los estudios de Palau-Sampio (2018), Vizoso y Vázquez-Herrero (2019) y Rodríguez-Pérez (2020), por su análisis de las tipologías de bulos es fundamental el trabajo de Salaverría et al (2020), y por las propuestas sobre curación los trabajos de López-Borrull con colaboradores.

Se ha visto que el fenómeno de la desinformación, para utilizar el término más general, es altamente poliédrico. Además de presentar diversas naturalezas y facetas, obedece a muy diversas motivaciones. Algunas son la simple parodia (aunque después puede generar desinformación en una parte del público), pero otras son la intención de causar daños personales 
o corporativos o de influir mediante engaños en procesos electorales, para citar algunos de los casos posiblemente más graves.

También se ha visto que, sin embargo, la sociedad tiene instrumentos para afrontar esta clase de pandemia: el periodismo de calidad, la educación mediática o la curación están entre los más destacados, a través de procedimientos como el periodismo de investigación, las actividades curatoriales, la verificación o el fact cheking.

Todo ello, sin dejar de ser importante, no libera a los ciudadanos de la obligación de ser socialmente responsables al menos en dos frentes. Por un lado, deben evitar convertirse en vectores de desinformación siendo razonablemente críticos a la hora de difundir noticias e informaciones. En segundo lugar, acudiendo únicamente a fuentes fiables (p.e., periodismo de medios de referencia y fuentes curadas de calidad).

En todo caso, serán necesarios estudios constantes sobre este tema crucial (sin exagerar) para el futuro de la humanidad.

\section{Agradecimientos}

Este trabajo forma parte del proyecto "Narración interactiva y visibilidad digital en el documental interactivo y el periodismo estructurado". RTI2018-095714-B-C21 (MICINN/FEDER), Ministerio de Ciencia, Innovación y Universidades (España) y ha contado con el apoyo del grupo de investigación consolidado "Cultura i Continguts Digitals" (SGR 2017-422), financiado por la Agència de Gestió d'Ajuts Universitaris i de Recerca (AGAUR) de la Generalitat de Catalunya (España).

\section{Referencias Bibliográficas}

Álvaro Sánchez, Sandra (2018). La esfera pública en la era de la hipermediación algorítmica: noticias falsas, desinformación y la mercantilización de la conducta. Hipertext.net, 17, pp. 74-82. https://dx.doi.org/10.31009/hipertext.net.2018.i17.07

American Psychological Association (2020). Publication Manual of the American Psychological Association. APA, USA.

Amorós García, Marc (2018). Fake News. La verdad de las noticias falsas. Plataforma Editorial. España

Aparici, Roberto; García-Marín, David (coords.) (2019). La posverdad. Una cartografía de los medios, las redes y la política. Gedisa. España

Aparici, Roberto; García-Marín, David; Rincón-Manzano, Laura (2019). "Noticias falsas, bulos y trending topics. Anatomía y estrategias de la desinformación en el conflicto catalán". El profesional de la información, 28 (3). España, pp. 1-17 https://doi.org/10.3145/epi.2019.may.13

Bernal-Triviño, Ana; Clares-Gavilán, Judith (2019). "Uso del móvil y las redes sociales como canales de verificación de fake news. El caso de Maldita.es". El profesional de la información, 28 (3). España, pp. 1-8. https://doi.org/10.3145/epi.2019.may.12

Blanco-Herrero, David; Carlos Arcila-Calderón. (2019). "Deontología y noticias falsas: estudio de las percepciones de periodistas españoles". El profesional de la información, 28 (3). España, pp. 1-13. https://doi.org/10.3145/epi.2019.may.08

Canavilhas, João; Colussi, Juliana; Moura, Zita-Bacelar (2019). Desinformación en las elecciones presidenciales 2018 en Brasil: un análisis de los grupos familiares en 
WhatsApp. El profesional de la información, 28 (5). España, pp.1-9 https://doi.org/10.3145/epi.2019.sep.03

Capilla, Pablo (2019). "¿De qué hablamos cuando hablamos de posverdad? Análisis del término en siete diarios de calidad". El profesional de la información, 28 (3). https://doi.org/10.3145/epi.2019.may.09

Caridad-Sebastián, Mercedes; Morales-García, Ana-María; Martínez-Cardama, Sara; GarcíaLópez, Fátima (2018). Infomediación y posverdad: el papel de las bibliotecas. EI profesional de la información, 27 (4), España, pp. 891-898.

https://doi.org/10.3145/epi.2018.jun.17

Castillo-Esparcia, Antonio; Fernández-Souto, Ana-Belén; Puentes-Rivera, Iván (2020). Comunicación política y Covid-19. Estrategias del Gobierno de España. Profesional de la información, 29 (4). https://doi.org/10.3145/epi.2020.jul.19

Catalán-Matamoros, Daniel (2020). La comunicación sobre la pandemia del COVID-19 en la era digital: manipulación informativa, fake news y redes sociales. RECS. Revista Española de Comunicación en Salud, suplemento 1 (pp. 1-4). https://doi.org/10.20318/recs.2020.5531

Codina, Lluís; Guallar, Javier (2019). Cura de continguts i fonts d'informació obertes per a comunicadors: relacions estratègiques en un periodisme de qualitat. Comunicació. $\begin{array}{llllll}\text { Revista de recerca i d'anàlisi, } 36 & \text { (2), pp. }\end{array}$ https://doi.org/10.2436/20.3008.01.185

Del-Fresno-García, Miguel (2019). Desórdenes informativos: sobreexpuestos e infrainformados en la era de la posverdad. El profesional de la información, 28 (3), e280302. https://doi.org/10.3145/epi.2019.may.02

Echevarría, Borja (2017). Más 'fact-checking' contra la posverdad. Cuadernos de periodistas, 33, pp. 9-16. http://www.cuadernosdeperiodistas.com/mas-fact-checking-laposverdad/

European Commission (2018). A multi-dimensional approach to disinformation. Report of the independent High Level Group on Fake News and Online Disinformation. Directorate-General for Communication Networks, Content and Technology. Union Europea. http://bit.ly/2Ponxc1

Facebook (2020). Centro de Información sobre el Coronavirus COVID-19. https://www.facebook.com/coronavirus info

García-Marín, David (2020). Infodemia global. Desórdenes informativos, narrativas fake y factchecking en la crisis de la Covid-19. Profesional de la información, 29 (4), e290411. https://doi.org/10.3145/epi.2020.jul.11

Guallar, Javier (2011). La documentación en la prensa digital. Nuevas tendencias y perspectivas. III Congreso Internacional de Ciberperiodismo y Web 2.0. Bilbao. Noviembre 2011. http://eprints.rclis.org/16326/

Guallar, Javier (2018). Prensa digital en 2015-2017. Los medios frente a las plataformas tecnológicas. Anuario ThinkEPI, 12, pp. 225-229. https://doi.org/10.3145/thinkepi.2018.33

Guallar, Javier; Codina, Lluís (2018). "Journalistic content curation and news librarianship: Differential characteristics and necessary convergence". El profesional de la información, 27 (4), pp. 778-791. https://doi.org/10.3145/epi.2018.jul.07 
Guallar, Javier; Cornet, Anna (2020). Centros de documentación de diarios en el siglo XXI. Panorama después del tsunami. BiD, n. 44. https://dx.doi.org/10.1344/BiD2020.44.6 Ireton, Cherilyn; Posetti, Julie (eds) (2018). Journalism, fake news \& disinformation. United Nations Educational, Scientific and Cultural Organization, France.

López-Borrull, Alexandre; Ollé, Candela (2019). La curación de contenidos científicos como respuesta a las noticias y a la ciencia falsas. Anuario ThinkEPI, 13, e13e07. España. https://doi.org/10.3145/thinkepi.2019.e13e07

López-Borrull, Alexandre; Vives-Gràcia, Josep; Badell, Joan-Isidre (2018). Fake news, ¿amenaza u oportunidad para los profesionales de la información y la documentación? El profesional de la información, 27, 6, España, pp. 1346-1356. https://doi.org/10.3145/epi.2018.nov.17

López Pan, Fernando; Rodríguez Rodríguez, Jorge (2020). El Fact Checking en España. Plataformas, prácticas y rasgos distintivos. Estudios sobre el mensaje periodístico, 26 (3), España, pp. 1045-1065. https://doi.org/10.5209/esmp.65246

Lotero-Echeverri, Gabriel, Romero-Rodríguez, Luis Miguel, Pérez-Rodríguez, Amor (2018). "Fact-checking vs. Fake news: Periodismo de confirmación como recurso de la competencia mediática contra la desinformación". Index. comunicación, 8 (2), pp. 295-

316. https://journals.sfu.ca/indexcomunicacion/index.php/indexcomunicacion/article/vi ew/370/400

Magallón-Rosa, Raúl (2018a). La biblioteca digital sobre Donald Trump. Fact-checking frente a fake news. Estudios sobre el Mensaje Periodístico, 24 (1), España, pp. 273282. https://doi.org/10.5209/ESMP.59949

Magallón-Rosa, Raúl (2018b). Nuevos formatos de verificación. El caso de Maldito Bulo en Twitter. Sphera publica, 18 (1), pp. 41-65. http://sphera.ucam.edu/index.php/sphera01/article/view/341

Magallón-Rosa, Raúl (2019). Verificado México 2018: Desinformación y fact-checking en campaña electoral. Revista de Comunicación, 18 (1), México, pp. 234-258. https://doi.org/10.26441/rc18.1-2019-a12

Marcos Recio, Juan Carlos; Sánchez Vigil, Juan Miguel; Olivera Zaldúa, María (2017). La enorme mentira y la gran verdad de la información en tiempos de la postverdad. Scire. 23 (2), España, pp.13-23.

https://www.ibersid.eu/ojs/index.php/scire/article/view/4446/3896

Martínez-Cardama, Sara; Algora-Cancho, Laura (2019). Lucha contra la desinformación desde las bibliotecas universitarias. El profesional de la información, 28 (4), España, e280412 https://doi.org/10.3145/epi.2019.jul.12

Marzal Felici, Javier y Casero Ripollés, Andreu (2017). Editorial. El fotoperiodismo en la era de la posverdad. En: adComunica. Revista Científica de Estrategias, Tendencias e Innovación en Comunicación, n¹3. Castellón: Asociación para el Desarrollo de la Comunicación adComunica y Universitat Jaume I, 11-17. DOI: http://dx.doi.org/10.6035/2174-0992.2017.13.1

Mayoral, Javier; Parratt, Sonia; Morata, Montserrat (2017). Desinformación, manipulación y credibilidad periodísticas: una perspectiva histórica. Historia y comunicación social, v. 24, n. 2, pp. 395-409. https://doi.org/10.5209/hics.66267 
Molina-Cañabate, Juan Pedro; Magallón-Rosa, Raúl (2019). Procedimientos para verificar y desmontar informaciones falsas basadas en el discurso del odio. El caso de Maldita Migración. RAEIC, Revista de la Asociación Española de Investigación de la Comunicación, 6 (12), España, pp. 95-122. http://doi.org/dh9n

Molina-Cañabate, Juan Pedro; Magallón-Rosa, Raúl (2020). Desinformación y periodismo científico. El caso de Maldita Ciencia. Revista Mediterránea de Comunicación/Mediterranean Journal of Communication, 11 (2), España, pp. 1121. https://www.doi.org/10.14198/MEDCOM2020.11.2.4

Nigro, Patricia (2018). Causas de la pérdida de la confianza en la prensa y estrategias para su restablecimiento en un contexto de incertidumbre. Hipertext.net, 17, pp. 54-63. https://doi.org/10.31009/hipertext.net.2018.117.0

Oxford Dictionary (2019). Fake news. https://oed.com/view/Entry/67776\#eid1264306660

Oxford University Press (2016). Word of the Year 2016 is... Oxford dictionaries. http://bit.ly/2BzsaJf

Palomo, Bella; Sedano, Jon (2018). WhatsApp como herramienta de verificación de fake news. El caso de B de Bulo. Revista Latina de Comunicación Social, 73, pp. 1384-1397. http://www.revistalatinacs.org/073paper/1312/71es.html

Palau-Sampio, Dolors (2018). Fact-checking y vigilancia del poder: La verificación del discurso público en los nuevos medios de América Latina. Communication \& Society, 31 (3), 347-365. https://doi.org/10.15581/003.31.3.347-365

Panetta, Kasey (2017). Gartner top strategic predictions for 2018 and beyond. Smarter with Gartner, October 3. Gartner. USA.

https://www.gartner.com/smarterwithgartner/gartner-top-strategic-predictions-for2018-and-beyond/

Parra Valero, Pablo; Oliveira, Lídia (2018). Fake news: una revisión sistemática de la literatura. Observatorio (OBS*), número especial, 54-78.

https://doi.org/10.15847/obsOBS12520181374

Posetti, Julie; Bontcheva, Kalina (2020a). Desinfodemia. Descifrando la desinformación sobre el COVID-19. Policy Brief 1. UNESCO. https://en.unesco.org/sites/default/files/disinfodemic_deciphering_covid19_disinforma tion_es.pdf

Posetti, Julie; Bontcheva, Kalina (2020b). Desinfodemia. Disección de las respuestas a la desinformación sobre el COVID-19. Policy Brief 2. UNESCO. https://en.unesco.org/sites/default/files/disinfodemic_dissecting_responses_covid19. disinformation es.pdf

Pozo-Montesa, Yaiza; León-Manove, Marina (2020). Plataformas fact-checking: las fakes news desmentidas por Newtral en la crisis del coronavirus en España. RECS. Revista Española de Comunicación en Salud, suplemento 1 (pp. 113-116). https://doi.org/10.20318/recs.2020.5446

Ramón Fernández, Francisca (2020). Comunicación y noticias falsas en relación al COVID-19: algunas reflexiones sobre la información, la desinformación y propuestas de mejora. RECS. Revista Española de Comunicación en Salud, suplemento 1 (pp. 253-264). https://doi.org/10.20318/recs.2020.5375 
Redondo, Myriam (2018). Verificación digital para periodistas. Manual contra bulos y desinformación internacional. UOC. España.

Rodrigo-Alsina, Miquel; Cerqueira, Laerte (2019). Periodismo, ética y posverdad. Cuadernos.Info, (44), pp. 225-239. https://doi.org/10.7764/cdi.44.1418

Rodríguez-Fernández, Leticia (2019). Desinformación: retos profesionales para el sector de la comunicación. El profesional de la información, 28 (3), España, e280306. https://doi.org/10.3145/epi.2019.may.06

Rodríguez-Ferrándiz, Raúl (2019). Posverdad y fake news en comunicación política: breve genealogía. El profesional de la información, 28 (3). https://doi.org/10.3145/epi.2019.may.14

Rodríguez-Pérez, Carlos (2019). No diga fake news, di desinformación: una revisión sobre el fenómeno de las noticias falsas y sus implicaciones. Comunicación, 40, pp. 65-74. https://doi.org/10.18566/comunica.n40.a05

Rodríguez-Pérez, Carlos (2020). Una reflexión sobre la epistemología del fact-checking journalism: retos y dilemas. Revista de Comunicación, 19 (1), España, pp. 243-258. https://doi.org/10.26441/RC19.1-2020-A14

Salaverría, Ramón; Buslón, Nataly; López-Pan, Fernando; León, Bienvenido; López-Goñi, Ignacio; Erviti, María-Carmen (2020). Desinformación en tiempos de pandemia: tipología de los bulos sobre la Covid-19. El profesional de la información, 29 (3), España, e290315. https://doi.org/10.3145/epi.2020.may.15

Sánchez-Duarte, José Manuel; Magallón-Rosa, Raúl (2020). Infodemia y COVID-19. Evolución y viralización de informaciones falsas en España. RECS. Revista Española de Comunicación en Salud, suplemento 1 (pp. 31-41). https://doi.org/10.20318/recs.2020.5417

Sánchez de la Nieta Hernández, Miguel Ángel; Fuente Cobo, Carmen (2020). Periodismo vs desinformación: la función social del periodista profesional en la era de las fake news y la posverdad. Sintaxis, 1 (4), México. Pp. 1-18.

https://doi.org/https://doi.org/10.36105/stx.2020n4.01

Seibt, Taís (2020). Limites e possibilidades do fact-checking como prática social para aperfeiçoar a democracia: o Truco nos Estados nas eleições brasileiras de 2018. Index.comunicación, $10 \quad$ (2), España, pp. 115-141. https://journals.sfu.ca/indexcomunicacion/index.php/indexcomunicacion/article/view/4 $\underline{51}$

Ufarte-Ruiz, María-José; Anzera, Guisseppe; Murcia-Verdú, Francisco-José (2020). Plataformas independientes de fact-checking en España e Italia. Características, organización y método. Revista Mediterránea de Comunicación, 11 (2), España, pp. 23-39. https://doi.org/10.14198/MEDCOM2020.11.2.3.

Ufarte-Ruiz, María José; Galletero-Campos, Belén; López-Cepeda, Ana María (2020). FactChecking, a Public Service Value in the Face of the Hoaxes of the Healthcare Crisis. Trípodos, 47, (1). España, Pp. 1-18

http://www.tripodos.com/index.php/Facultat_Comunicacio_Blanquerna/article/view/805/824

Ufarte-Ruiz, María José; Murcia-Verdú, Francisco José (2018). El fact checking: en busca de un nuevo modelo de negocio sostenible para el periodismo. Estudio de caso de Miniver. Miguel Hernández Communication Journal, 9 (2), pp. 511534. http://dx.doi.org/10.21134/mhcj.v0i9.26 
Ufarte-Ruiz, María José, Peralta-García, Lidia; Murcia-Verdú, Francisco José (2018). Fact checking: un nuevo desafío del periodismo. El profesional de la información, 27 (4), España, pp. 733-741. https://doi.org/10.3145/epi.2018.jul.02

Vázquez-Herrero, Jorge; Vizoso, Ángel; López-García, Xosé (2019). Innovación tecnológica y comunicativa para combatir la desinformación: 135 experiencias para un cambio de rumbo. El profesional de la información, 28 (3), España, e280301. https://doi.org/10.3145/epi.2019.may.01

Vizoso, Ángel; Vázquez-Herrero, Jorge (2019). Plataformas de fact checking en español. Características, organización y método. Communication \& Society, 32 (1), España, pp. 127-144. https://doi.org/10.15581/003.32.1.127-144

Wardle, Claire; Derakhshan, Hossein (2017). Information disorder: toward an interdisciplinary framework for research and policy making. Council of Europe. Francia.

WHO (2020). Novel Coronavirus (2019-nCoV) Situation Report - 13. World Health Organizarion, 2 February. https://www.who.int/docs/defaultsource/coronaviruse/situation-reports/20200202-sitrep-13-ncov-v3.pdf 